\title{
An aberrant venous channel mimicking the perilabyrinthine cells in the petrous bone of a patient with vestibular schwannoma: illustrative case
}

\author{
Masato Ito, MD, ${ }^{1}$ Yoshinori Higuchi, MD, ${ }^{1}$ Kentaro Horiguchi, MD, ${ }^{1}$ Shigeki Nakano, MD, ${ }^{1}$ Shinichi Origuchi, MD, ${ }^{1}$ Kyoko Aoyagi, MD, ${ }^{2}$ \\ Toru Serizawa, MD, ${ }^{3}$ Iwao Yamakami, MD, ${ }^{4}$ and Yasuo Iwadate, $\mathrm{MD}^{1}$ \\ ${ }^{1}$ Department of Neurological Surgery, Chiba University Graduate School of Medicine, Chiba, Japan; ${ }^{2}$ Department of Neurosurgery, Chiba Cerebral and Cardiovascular Center, \\ Ichihara, Japan; ${ }^{3}$ Tokyo Gamma Unit Center, Tsukiji Neurological Clinic, Tokyo, Japan; and ${ }^{4}$ Department of Neurosurgery, Seikeikai Chiba Medical Center, Chiba, Japan
}

\begin{abstract}
BACKGROUND Anatomical variations, such as high jugular bulbs and air cell development in the petrosal bone, should be evaluated before surgery. Most bone defects in the internal auditory canal (IAC) posterior wall are observed in the perilabyrinthine cells. An aberrant vascular structure passing through the petrous bone is rare.

OBSERVATIONS A 48-year-old man presented with a right ear hearing disturbance. Magnetic resonance imaging revealed a 23-mm contrastenhancing mass in the right cerebellopontine angle extending into the IAC, consistent with a right vestibular schwannoma. Preoperative bone window computed tomographic scans showed bone defects in the IAC posterior wall, which ran farther posteroinferiorly in the petrous bone, reaching the medial part of the jugular bulb. The tumor was accessed via a lateral suboccipital approach. There was no other major vein in the cerebellomedullary cistern, except for the vein running from the brain stem to the IAC posterior wall. To avoid complications due to venous congestion, the authors did not drill out the IAC posterior wall or remove the tumor in the IAC.
\end{abstract}

LESSONS Several aberrant veins in the petrous bone are primitive head sinus remnants. Although rare, their surgical implication is critical in patients with vestibular schwannomas.

https://thejns.org/doi/abs/10.3171/CASE21487

KEYWORDS vestibular schwannoma; aberrant vein channel; petrous bone; venous complication

Drilling the internal auditory canal (IAC) posterior wall is crucial in removing a vestibular schwannoma. Anatomical variations, such as high jugular bulbs (JBs) and air cell development in the petrosal bone, should be evaluated before surgery. ${ }^{1}$ Perilabyrinthine cells in the petrous bone are exposed during drilling of the IAC posterior part and can cause postoperative cerebrospinal fluid leakage after inappropriate repair procedures.

Aberrant vascular structures passing through the petrous bone are rare. These vessels might be related to the vascular structure variation formed during the temporal bone ossification during head embryogenesis. Several aberrant veins in the petrous bone are the primitive head sinus (primary capital vein) remnants. ${ }^{2,3}$ Herein, we report a case of an aberrant vein passing through the IAC posterior wall with superior petrosal vein (SPV) and superior petrosal sinus (SPS) aplasia. The abnormal vein was asymptomatic; however, its surgical relevance is critical in patients with vestibular schwannomas.

\section{Illustrative Case}

A 48-year-old man presented with a right ear hearing disturbance that he had experienced for 3 years and a hearing disturbance at a higher frequency that had progressed slowly. Magnetic resonance (MR) images revealed a 23-mm contrast-enhancing mass in the right cerebellopontine angle extending into the internal auditory meatus, consistent with a right vestibular schwannoma (Fig. $1 \mathrm{~A}$ and $\mathrm{B}$ ). Audiometry revealed

ABBREVIATIONS 3D-CTA = three-dimensional computed tomography angiography; $\mathrm{AICA}=$ anterior inferior cerebellar artery; $\mathrm{CT}=$ computed tomography; DP = dural plexus; IAC = internal auditory canal; JB = jugular bulb; $M R=$ magnetic resonance; $P H S=$ primary head sinus; SA = subarcuate artery; SPS = superior petrosal sinus; SPV = superior petrosal vein.

INCLUDE WHEN CITING Published November 1, 2021; DOI: 10.3171/CASE21487.

SUBMITTED August 27, 2021. ACCEPTED September 16, 2021.

(C) 2021 The authors, CC BY-NC-ND 4.0 (http://creativecommons.org/licenses/by-nc-nd/4.0/). 

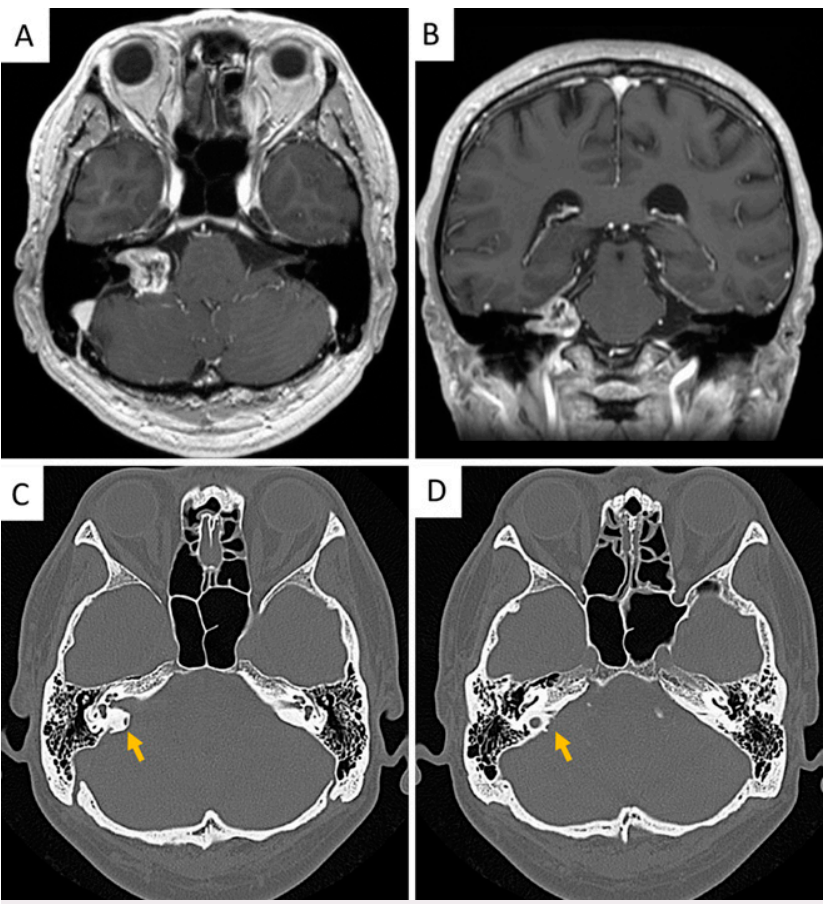

FIG. 1. Axial (A) and coronal (B) MR images with gadolinium enhancement reveal a 23-mm-diameter tumor extending into the right cerebellopontine cistern. Bone window CT scans show bone defects in the IAC posterior wall (C) extending further posteroinferiorly in the petrous bone (D) (arrows).

serviceable hearing with a pure-tone average of $30 \mathrm{~dB}$ and a speech discrimination score of $90 \%$ in the right ear. Preoperative bone window computed tomography (CT) scans showed bone defects on the IAC posterior wall, which ran farther posteroinferiorly in the petrous bone, reaching the medial part of the JB (Fig. $1 \mathrm{C}$ and D). Three-dimensional CT angiography (3D-CTA) for preoperative assessment of the venous phase showed neither the right SPS nor the SPV (Fig. 2A-D). A vein ran from the brain stem to the petrosal bone close to the IAC and reached the JB (Fig. 2D). The patient underwent tumor removal with hearing preservation.

The tumor was accessed via a lateral suboccipital approach to monitor the cochlear nerve action potential at the park bench position. During tumor dissection, the SPV was not identified. There was no other major vein in the cerebellomedullary cistern, except the vein running from the brain stem to the IAC posterior wall (Fig. 3A). We concluded that the aberrant vein needed posterior fossa venous drainage and was essential for the patient. To avoid complications due to venous congestion, we did not drill out the IAC posterior wall or remove the tumor in the IAC (Fig. 3B). The patient's postoperative course was uneventful. Audiometry revealed a pure-tone average of $33.8 \mathrm{~dB}$, and the serviceable hearing status was preserved (American Academy of Otolaryngology-Head and Neck Surgery class B) 6 months after the surgery. Postoperative MR imaging revealed that the cisternal portion's tumor was removed, and the flow of the aberrant vein was preserved (Fig. 4A-D). The residual tumor was treated with stereotactic radiosurgery (12 Gy) at the periphery 6 months after the surgery.
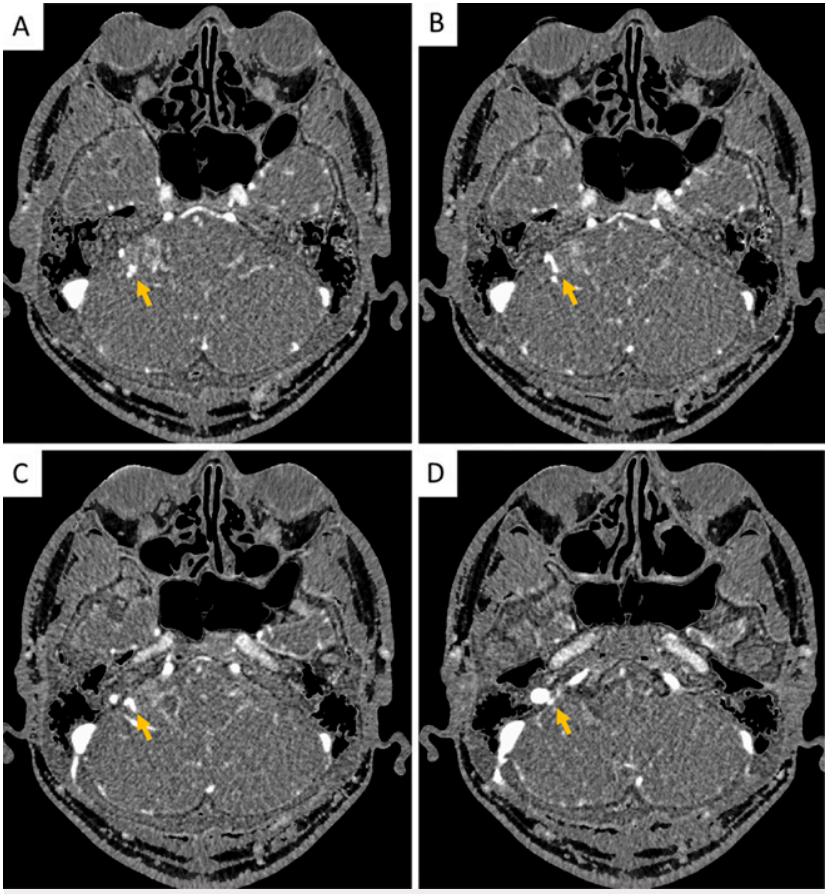

FIG. 2. 3D-CTA in venous phase before surgery reveals right superior petrosal sinus and vein aplasia. Arrows indicate that an aberrant vessel runs through the right cerebellopontine cistern $(\mathbf{A}-\mathbf{C})$ and reaches the right JB (D). Levels of the images (A-D) are in the rostral to caudal direction.

\section{Discussion \\ Observations}

We encountered a case of an aberrant venous channel in the petrous bone of a patient with underdeveloped SPS and SPV. The bone defect mimicked perilabyrinthine cells in the petrous bone. The channel seemed essential for the posterior fossa venous circulation based on the operative findings. The underdeveloped SPS and SPV is a unique variant because it is one of the most stable and largest venous complexes in the posterior fossa. ${ }^{4}$ The SPV and SPS are occasionally absent, and the tributaries of the galenic and tentorial groups perform the venous drainage in these circumstances. ${ }^{4}$ Our patient did not have another venous drainage pathway other than the aberrant venous channel. There was no direct evidence that the anomalous vein is the essential venous drainage pathway of the right posterior fossa in the present patient; however, we could not locate other main venous drainages from the right cerebellum on the 3D-CTA scan.

\section{Normal SPV and SPS Development}

The development of the venous drainage of the embryonic cranial structures is a complex phenomenon. ${ }^{5}$ The main streams of venous return of the brain stem in human embryos at 4 weeks are positioned diagonally. Primitive vascular loops form on the surface of the mesencephalon, metencephalon, and myelencephalon longitudinally and transversely. Primitive transverse veins drain into the dural plexus (DP), the origin of the dural venous sinus. The DP drains into a longitudinal channel, its cephalic portion originating within the head and its caudal portion belonging to the cardinal system. This entire channel is called the "primary head sinus" (PHS). The PHS is the terminal endocranial venous 

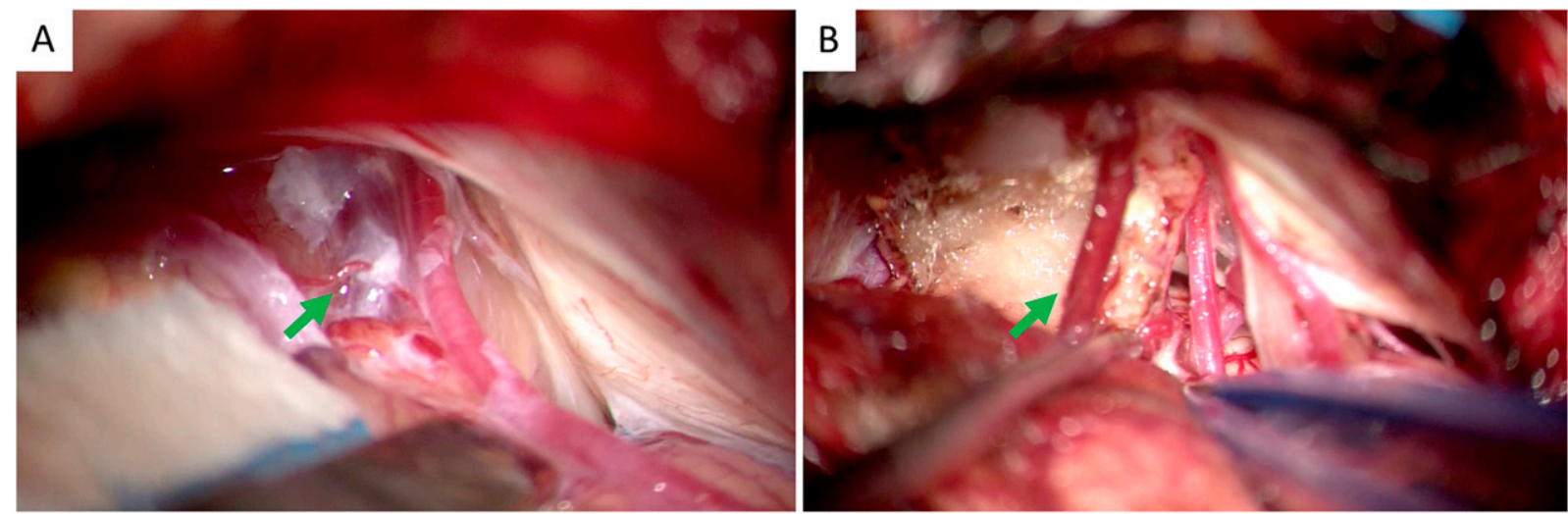

FIG. 3. Intraoperative photos demonstrate an aberrant vein (green arrow) running from the brain stem to the IAC posterior wall (A). This vein is preserved after tumor removal $(\mathbf{B})$.

structure that returns to the anterior cardinal vein, and its channel is outside the otic capsule, which develops into the auditory organs. The tendency toward lateral migration from the development of the otic capsule causes the PHS to divide and disappear in a 5-week human embryo. ${ }^{6}$ Primitive transverse and sigmoid sinuses form surrounding the otic capsule during its development, and anastomoses develop between them in the 6-week human embryo. ${ }^{6}$

The SPS is the last of the major adult sinuses to be formed. It is derived from the ventral metencephalic vein, ${ }^{6}$ or a small plexiform tributary of the prootic sinus, ${ }^{7}$ related to early cerebellum development. A PHS-derived remnant vein sometimes sustains at the cranial and caudal sides. The caudal side region remains around the dural end of the ventral myelencephalic vein, which joins the internal jugular vein below
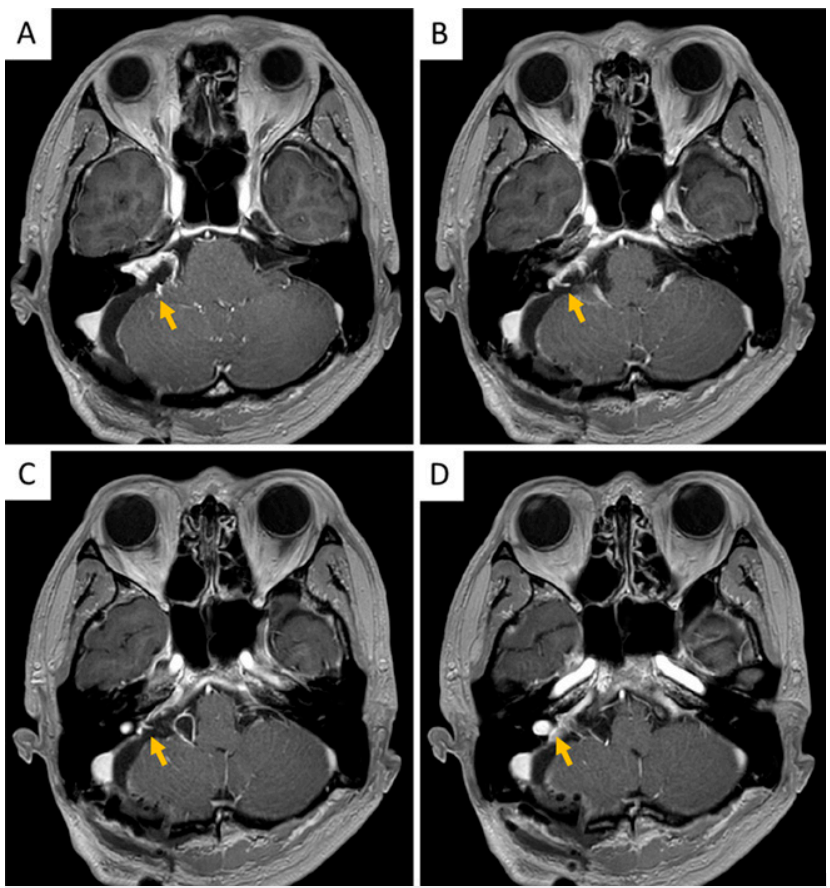

FIG. 4. Postoperative MR imaging shows that the cisternal portion's tumor is removed (arrows) (A-D). its foramen. ${ }^{6}$ With the PHS disappearance, most primary transverse veins disappear; however, limited primitive transverse veins remain along the cranial nerves. ${ }^{6}$ The normal SPV is composed of the assimilation of remnant primitive transverse veins.

\section{Literature Review of Aberrant Veins in the Petrous Bone}

The petrous bone has complicated anatomy. Complex bony structures, small canals, and fine sutures sometimes facilitate differentiation between the normal and abnormal formations. A persistent stapedial artery, aberrant internal carotid artery, dehiscent JB, high JB with diverticulum, and anterior and dehiscent sigmoid sinuses have been detected in $<1 \%$ of the temporal bones. ${ }^{8}$ Several reports have demonstrated an aberrant vascular channel in the petrous bone., ${ }^{2,9}$ Hermans and van Rensburg $^{2}$ reported four patients with an aberrant vascular channel in the petrous bone and concluded that these anomalous vessels are derived from the lateral capital vein remnants, the caudal PHS. The aberrant vascular channel originated close to the sulcus of the SPS. Three of the four patients had only CT scans without enhanced MR images, which confirmed that the channel in the petrous bone had a vascular structure. This channel was connected between the intracranial veins and the JB in two cases, and these pathways were similar to those in our case. A deviant channel may also form during the otic capsule development.

Mizutani et al. ${ }^{9}$ reported remnant PHS in the case of dural arteriovenous fistula. The deviant vein ran from the middle fossa to the JB and penetrated the petrous bone ventrolaterally to reach the inner ear and internal carotid artery. The authors considered the aberrant channel a PHS remnant for the following reasons: The channel's position was lateral to the cranial nerves (7th-12th) and inferolateral to the labyrinth and the inner ear, the predictive course of a PHS remnant. The authors suggested that the intraosseous vascular channels were formed before the petrous bone ossification. We could not directly verify if the aberrant vascular path in the petrous bone was PHS derived. However, on the basis of anatomical localization, we suggest that the anomalous vein was PHS derived during embryogenesis.

\section{Origin of the Aberrant Vein}

The vascular channel observed in our case did not correspond to the localization of the temporal bone emissary veins, like either the mastoid and condylar emissary veins or the petrosquamosal veins. The primary transverse veins and the DP involve normal 
SPS and SPV development (Fig. 5A and B). The SPS aplasia suggests embryological participation in the aberrant venous pathway development. The deviant venous channel in the petrous bone might have formed during early embryogenesis (Fig. $5 \mathrm{C}$ ). In the absence of a venous drainage pathway through the SPS, a DP remnant, the PHS, and metencephalic vein formed an aberrant venous pathway in the present patient (Fig. 5D).

\section{Significance of the Vessels Proximal to the IAC}

Most anatomical variations in the aberrant vascular pathway have nonpathological relevance. However, some of them have the potential for surgical significance. The IAC posterior wall should be drilled to open the IAC for the vestibular schwannoma resection. Total removal with minimum morbidity can be achieved following the IAC posterior wall, ${ }^{10,11}$ and IAC tumor removal is critical to prevent residual tumor recurrence or regrowth. ${ }^{12}$

The subarcuate artery (SA) enters the subarcuate canal that originates from the subarcuate fossa proximal to the IAC. It typically supplies the otic capsule of the semicircular canals, vestibule, facial nerve canal, and mastoid antrum. ${ }^{13,14}$ It ends in the subarcuate fossa dura mater and is present in only $35 \%$ of cases. ${ }^{15} \mathrm{~A}$ short $\mathrm{SA}$ could be surgically challenging due to anterior inferior cerebellar artery (AICA) anchoring to the petrous surface, ${ }^{16}$ however, SA sacrifice causes no neurologic deficits. ${ }^{14,16}$

Anomalous vascular anatomy within the cerebellopontine angle increases the difficulty of tumor removal. Attention has been paid to the vascular loop embedded in the IAC posterior wall. The presence of this loop creates the potential for technical difficulties and major complications. Previous reports described the incidence of the AICA embedded in the subarcuate fossa as $1 \%-6.7 \%{ }^{16-18}$ Warren et al. ${ }^{18}$ demonstrated that $12.5 \%$ of these patients experienced significant morbidity after tumor removal. Yamakami et al. ${ }^{16}$ postulated the anomalous vascular loop of the AICA proximal to the IAC as a "challenging AICA." The challenging AICA requires attention to procedures surrounding the IAC to avoid AICA injury.

The aberrant vessel in our case might be scarce because few reports have demonstrated anomalous venous channels penetrating the dura and petrous bone. Most of these aberrant vascular channels are asymptomatic. However, the surgical relevance of the vascular channel in the IAC posterior wall is more obvious in patients with
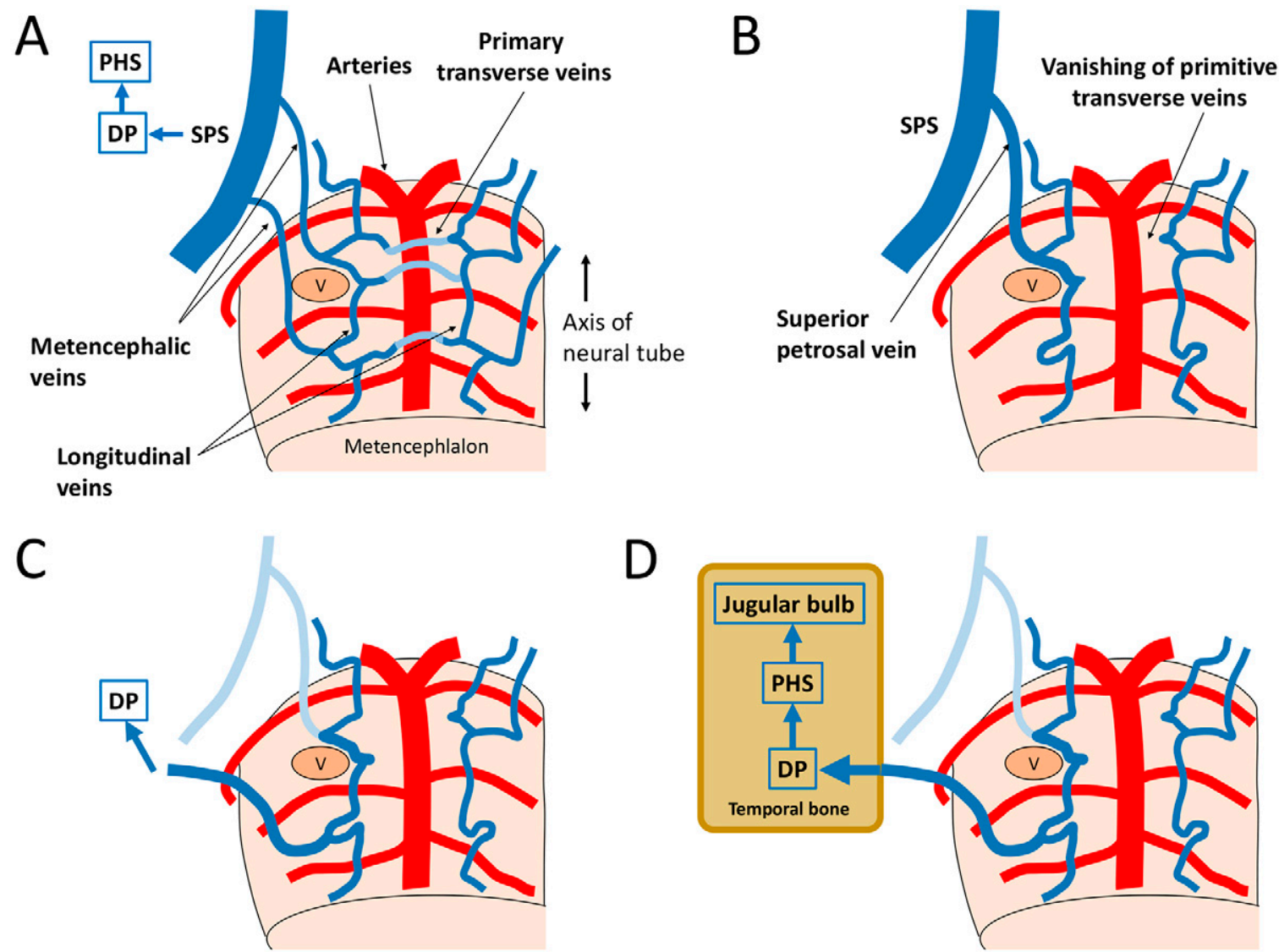

FIG. 5. Normal SPV development ( $A$ and $B$ ) and SPV and SPS aplasia ( $C$ and D). V indicates the trigeminal nerve root entry portion. A: Metencephalon and SPS ventral view. Metencephalic veins receive venous drainage from the metencephalon via the primitive transverse and longitudinal veins. Venous drainage pathway reaches through the SPS, DP, and subsequently the PHS. B: After the disappearance of the primitive transverse veins, DP, and PHS, SPV is developed from the metencephalic vein. C: SPS and ventral metencephalic vein aplasia. Another metencephalic vein close to the IAC receives venous drainage from the ventral metencephalon. D: Venous drainage via the aberrant vein runs into the DP, PHS, and JB in the temporal bone. 
vestibular schwannoma, especially in combination with other vascular variations, such as SPV and SPS hypoplasia or aplasia.

We decided to preserve the aberrant vein for the following reasons: (1) We did not identify the venous pathway of the cerebellar petrous surface, and (2) our patient did not have a well-developed collateral drainage through the galenic and tentorial tributaries, crucial venous pathways when either the SPS or SPV is aplastic. ${ }^{4}$ Narayan et al. ${ }^{19}$ reported a systematic review of the safety profile of SPV sacrifice in neurosurgical procedures. The review suggests that SPV obliteration may be associated with a $0.2 \%$ complication rate. ${ }^{19}$ Therefore, it can be presumed that most SPV obliteration cases are not related to a high incidence of venous complications. However, the collateral pathway was not analyzed in this systematic review. We could not verify the importance of the aberrant vein in the present case; however, venous drainage from the cerebellar petrous surface possibly depended on the anomalous vein passing through the petrous bone in this case.

\section{Treatment Options for Patients With the Rare Anomalous Vein}

Patients with small to medium vestibular schwannomas are good candidates for Gamma Knife radiosurgery. When resection is not chosen by the patient, early SRS intervention, in contrast to observation, results in long-term tumor control and improved rates of hearing preservation. ${ }^{20}$ Furthermore, small tumor volume $\left(<1.2 \mathrm{~cm}^{3}\right)$, younger age $(<45$ years old), and Gardner-Robertson grade 1 hearing status are favorable predicting factors for preserving serviceable hearing status after Gamma Knife radiosurgery. ${ }^{21}$ Tumors $<2.5 \mathrm{~cm}$ in diameter are preferred for treatment using Gamma Knife radiosurgery to minimize the risk of radiation-induced edema, trigeminal neuropathy, hydrocephalus, and diminished long-term tumor control. ${ }^{22}$ We initially chose surgical resection to preserve the hearing status because the tumor size seemed too large for Gamma Knife radiosurgery to preserve functional hearing.

Several studies have demonstrated the efficacy of Gamma Knife radiosurgery as a first-line treatment for large vestibular schwannomas. ${ }^{23-25}$ Nevertheless, surgical resection remains the primary approach; long-term tumor control has been reported as 78.8\%-98.3\%, which is moderately acceptable in large vestibular schwannomas. ${ }^{23-25}$ The hearing preservation rate is $31.3 \%-65.8 \% .^{23-25}$ Hearing preservation remains challenging, and Gamma Knife radiosurgery can be considered an effective option, particularly in patients who are poor candidates for surgery. When extensive imaging, including vascular evaluation using 3D-CTA, detects an aberrant vein in the posterior lip of the IAC, without other major venous drainage routes in the ipsilateral posterior fossa, Gamma Knife radiosurgery can be an appropriate initial treatment option, considering the difficulty in surgically removing tumors in the IAC.

\section{Lessons}

Most bone defects in the IAC posterior wall are perilabyrinthine cells. Although rare, we should consider that we may encounter anatomical variations interfering with the surgical procedures in vestibular schwannoma.

\section{References}

1. Yamakami I, Uchino Y, Kobayashi E, Yamaura A. Computed tomography evaluation of air cells in the petrous bone - relationship with postoperative cerebrospinal fluid rhinorrhea. Neurol Med Chir (Tokyo). 2003;43(7):334-339.

2. Hermans R, van Rensburg LJ. An aberrant vascular channel in the petrous bone: persistent lateral capital vein? Eur Radiol. 2009;19(12): 2958-2964.
3. Mitsuhashi Y, Hayasaki K, Kawakami T, et al. Dural venous system in the cavernous sinus: a literature review and embryological, functional, and endovascular clinical considerations. Neurol Med Chir (Tokyo). 2016;56(6):326-339.

4. Matsushima K, Ribas ES, Kiyosue H, Komune N, Miki K, Rhoton $\mathrm{AL}$. Absence of the superior petrosal veins and sinus: surgical considerations. Surg Neurol Int. 2015;6:34.

5. Streeter GL. The development of the venous sinuses of the dura matter in the human embryo. Am J Anat. 1915;18(2):145-178.

6. Padget $\mathrm{DH}$. The cranial venous system in man in reference to development, adult configuration, and relation to the arteries. Am J Anat. 1956;98(3):307-355.

7. Butler $\mathrm{H}$. The development of certain human dural venous sinuses. J Anat. 1957;91(4):510-526.

8. Koesling S, Kunkel P, Schul T. Vascular anomalies, sutures and small canals of the temporal bone on axial CT. Eur J Radiol. 2005;54(3):335-343.

9. Mizutani K, Akiyama T, Yoshida K. The remnant of primary head sinus found in the case of dural arteriovenous fistula: a case report. Interv Neuroradiol. 2016;22(4):452-456.

10. Yamakami I, Uchino Y, Kobayashi E, Yamaura A, Oka N. Removal of large acoustic neurinomas (vestibular schwannomas) by the retrosigmoid approach with no mortality and minimal morbidity. J Neurol Neurosurg Psychiatry. 2004;75(3):453-458.

11. Yamakami I, Ito S, Higuchi Y. Retrosigmoid removal of small acoustic neuroma: curative tumor removal with preservation of function. $J$ Neurosurg. 2014;121(3):554-563.

12. Breshears JD, Morshed RA, Molinaro AM, McDermott MW, Cheung SW, Theodosopoulos PV. Residual tumor volume and location predict progression after primary subtotal resection of sporadic vestibular schwannomas: a retrospective volumetric study. Neurosurgery. 2020;86(3):410-416.

13. Mazzoni A. The subarcuate artery in man. Laryngoscope. 1970;80(1):69-79.

14. Grammatica A, Alicandri-Ciufelli M, Molteni G, Marchioni D, Presutti L. Subarcuate canal and artery: a case report. Surg Radiol Anat. 2010;32(2):171-174.

15. Salgado-Lopez L, Leonel LCP, Aydin SO, Peris-Celda M. Surgical anatomy of the labyrinthine and subarcuate arteries and clinical implications. World Neurosurg. 2020;141:e880-e887.

16. Yamakami I, Kubota S, Higuchi $Y$, Ito S. Challenging anterior inferior cerebellar artery in retrosigmoid vestibular schwannoma removal. World Neurosurg. 2019;121:e370-e378.

17. Tanriover N, Rhoton AL Jr. The anteroinferior cerebellar artery embedded in the subarcuate fossa: a rare anomaly and its clinical significance. Neurosurgery. 2005;57(2):314-319.

18. Warren DT, Warren MD, Malfair D, Akagami R. An incidence of anteroinferior cerebellar artery/posteroinferior cerebellar artery anatomic variants penetrating the subarcuate fossa dura: operative technique and identification with 3-dimensional fast imaging employing steady-state acquisition magnetic resonance imaging. Neurosurgery. 2010;66(6 Suppl Operative):199-204.

19. Narayan V, Savardekar AR, Patra DP, et al. Safety profile of superior petrosal vein (the vein of Dandy) sacrifice in neurosurgical procedures: a systematic review. Neurosurg Focus. 2018;45(1):E3.

20. Kondziolka D, Mousavi SH, Kano H, Flickinger JC, Lunsford LD. The newly diagnosed vestibular schwannoma: radiosurgery, resection, or observation? Neurosurg Focus. 2012;33(3):E8.

21. Johnson S, Kano H, Faramand A, Niranjan A, Flickinger JC, Lunsford $\mathrm{LD}$. Predicting hearing outcomes before primary radiosurgery for vestibular schwannomas. J Neurosurg. 2020;133(4):1235-1241.

22. Carlson ML, Link MJ. Vestibular schwannomas. N Engl J Med. 2021;384(14):1335-1348.

23. van de Langenberg $R$, Hanssens PE, Verheul JB, et al. Management of large vestibular schwannoma. Part II. Primary Gamma Knife surgery: radiological and clinical aspects. J Neurosurg. 2011;115(5):885-893. 
24. Lefranc M, Da Roz LM, Balossier A, Thomassin JM, Roche PH, Regis J. Place of Gamma Knife stereotactic radiosurgery in grade 4 vestibular schwannoma based on case series of 86 patients with long-term follow-up. World Neurosurg. 2018;114:e1192-e1198.

25. Bailo M, Boari N, Franzin A, et al. Gamma Knife radiosurgery as primary treatment for large vestibular schwannomas: clinical results at long-term follow-up in a series of 59 patients. World Neurosurg. 2016;95:487-501.

\section{Disclosures}

The authors report no conflict of interest concerning the materials or methods used in this study or the findings specified in this paper.

\section{Author Contributions}

Conception and design: Higuchi, Aoyagi, Serizawa, Iwadate. Acquisition of data: Higuchi, Ito, Nakano, Origuchi, Aoyagi. Analysis and interpretation of data: Ito, Nakano, Aoyagi. Drafting the article: Higuchi, Ito, Aoyagi. Critically revising the article: Higuchi, Ito,
Nakano, Aoyagi, Serizawa, Yamakami. Reviewed submitted version of manuscript: Higuchi, Ito, Horiguchi, Nakano, Aoyagi, Serizawa, Yamakami. Approved the final version of the manuscript on behalf of all authors: Higuchi. Study supervision: Higuchi, Horiguchi, Iwadate.

\section{Supplemental Information}

Previous Presentations

Part of this paper was presented previously at the 30th Annual Meeting of the Japanese Society of Acoustic Neuroma, June 12, 2021 (virtual meeting).

\section{Correspondence}

Yoshinori Higuchi: Chiba University Graduate School of Medicine, Chiba, Japan. yhiguchi@faculty.chiba-u.jp. 